cases with concomitant LG and this could be used to suggest the diagnosis where no duodenal biopsy is supplied.

\section{P53 IN BARRETT'S OESOPHAGUS WITH CHANGES INDEFINITE FOR DYSPLASIA}

\author{
G. Miller $^{1,2}$, I. Brown ${ }^{1,2}$ \\ ${ }^{1}$ Envoi Specialist Pathologists, Brisbane, Australia; and \\ ${ }^{2}$ University of Queensland, Brisbane, Australia
}

Background: Barrett's oesophagus (BO) is a known precursor of dysplasia and adenocarcinoma of the oesophagus. Some cases of $\mathrm{BO}$ show changes in the epithelium equivocal for dysplasia and ancillary testing of these cases would be useful to help predict their malignant potential. P53 immunohistochemistry has been advocated as a possible marker for dysplasia in BO, however it is not well studied in cases with changes described as indefinite for dysplasia.

Aims: To determine whether the use of P53 immunohistochemistry predicts dysplasia on follow up biopsies from cases with an initial diagnosis of $\mathrm{BO}$ with changes indefinite for dysplasia.

Methods: Retrospective analysis of cases with a consensus diagnosis of $\mathrm{BO}$ with changes indefinite for dysplasia and at least one subsequent biopsy from the Barrett's segment. Patient age, sex and length of BO were recorded. P53 was recorded as either negative or positive based on strong staining of at least 1 crypt in the atypical area. This was then correlated with the diagnosis on subsequent biopsies.

Results and conclusions: 125 cases of BO indefinite for dysplasia were stained with P53. 19 cases showed strong positive staining and 104 cases showed weak background staining only. Strong P53 was seen in 9/19 (47\%) cases with high grade dysplasia on follow-up biopsy while strong P53 was only seen in $5 / 104(4.8 \%)$ of cases that did not show high grade dysplasia on follow-up $(p<0.0001)$. In conclusion, P53 may be of some use in determining which cases of $\mathrm{BO}$ with changes indefinite for dysplasia will not progress to high grade dysplasia.

\section{SCHISTOSOMIASIS MIMICKING INFLAMMATORY BOWEL DISEASE}

K. Kasem $^{1,2}$, J. Bradbear ${ }^{1,2}$, A. Lam ${ }^{1,2}$

${ }^{1}$ Griffith University, Gold Coast, Australia; and ${ }^{2}$ Gold Coast

University Hospital, Gold Coast, Australia

Background: Schistosomiasis is a parasitic disease with various clinical presentations caused by trematode blood flukes. Intestinal manifestations are not uncommon where the disease is prevalent. Schistosomiasis is however quite rare in Australia and is always imported. It is therefore rarely suspected, especially with atypical clinical manifestations.

Aims: The aim of this report is to illustrate the importance of this rare but treatable diagnostic entity, especially with vague unexplainable clinical picture.

Methods: We report a case of 60-year-old female who presented with 2 weeks of left lower quadrant abdominal pain and diarrhoea. The patient emigrated from Southern Philippines and had been living in Australia for two decades, with her last visit to the Philippines being 5 years ago. Background history included hypertension, urethral strictures and mild asthma. Physical examination was unremarkable while investigations revealed mild leukocytosis, eosinophilia and mildly elevated C-reactive protein and stool calprotectin.

CT scan showed rectosigmoid thickening and ulcer formation, suggestive of inflammatory bowel disease. Flexible sigmoidoscopy showed no significant abnormality. Multiple biopsies from the large bowel showed a large number of calcified parasitic eggs of Schistosoma, in keeping with chronic schistosomiasis. There was no clinical suspicion of schistosomiasis in this case and the biopsy diagnosis came as a surprise.

The patient was treated with praziquantel and follow up stool samples were clear. Symptoms of diarrhoea and abdominal pain resolved.

Conclusions: This is the first Australian case report describing schistosomiasis mimicking chronic inflammatory bowel disease presentation. Although very rare in Australia, schistosomiasis should be on the list of differential diagnoses of unexplained gastrointestinal symptoms.

\section{STRUMAL CARCINOID - WHEN TWO BECOME ONE}

E. Roper ${ }^{1}$, R. Chan ${ }^{1}$

${ }^{1}$ Concord Repatriation General Hospital, Sydney, Australia

Background: Strumal carcinoid is the rare coexistence of both struma ovarii and carcinoid. Recognition of this essentially benign entity avoids misdiagnosis and over-treatment.

Aims: To present the macroscopic, microscopic, immunohistochemical, and ultrastructural findings of a case of strumal carcinoid.

Methods: A 51-year-old female presented with a $4 \mathrm{~cm}$ left adnexal mass that was resected and received by the Anatomical Pathology Department at Concord Repatriation General Hospital. Macroscopic examination, light microscopy, immunohistochemistry, and electron microscopy were performed.

Results and conclusions: Macroscopically, the specimen consisted of a $40 \mathrm{~mm}$ cystic lesion lined by dark-brown granular material, with a $14 \mathrm{~mm}$ brown-and-spongy intramural nodule including a colloid-like area. By light microscopy, the cyst was a haemorrhagic corpus luteum and the nodule was a biphasic tumour composed of thyroid tissue and rosette-forming/trabecular areas. By immunohistochemistry, the rosette-forming/ trabecular areas were positive for CD56 and NSE-though negative for chromogranin and synaptophysin. By electron microscopy, the rosette-forming/trabecular areas contained 'saltand-pepper' nuclear chromatin and cytoplasmic single-membrane bound dense core granules. Therefore, the biphasic tumour was concluded to be composed of both thyroid and neuroendocrine tissue and a diagnosis of strumal carcinoid was made. Strumal carcinoid is the intimate association of thyroid and carcinoid tissue, seen in $25 \%$ of primary ovarian carcinoid. It is a unilateral lesion, 1-20 cm, beefy-red to yellow, and can be nearpure or part of a cystic teratoma. The morphologic and immunohistochemical features are predictable. The prognosis is almost invariably benign and local disease is treated by salpingooopherectomy, however a study of 50 cases reported one incident of peritoneal metastasis and death. In this same study, strumal carcinoid had been misdiagnosed as malignant transformation of struma ovarii in 4 of 50 cases. The diagnostic pitfall today is to over-interpret the frequently present mucinous epithelium of strumal carcinoid as representing mucinous (goblet cell) carcinoid. 